\title{
Performance Analysis of Wireless Local Area Networks
}

\author{
Muhammad Umair Naru, Umar Nauman and Khalid Hussain
}

\begin{abstract}
In an infrastructure based wireless network, access point (AP) is the central authority which controls all the registered nodes and relaying traffic in the network. Even though all traffic is routed through AP, the open nature of the underlying medium is prone to environmental factors, etc. which degrade the performance of the overall network. However, in a saturated case the AP becomes the bone of contention among nodes which can result in collisions, packet drops, etc.

In this study, authors analyze the performance of 802.11 WLANs that utilizes the Distributed Coordination Function (DCF). A simulation study having contending stations within the transmission range of an $\mathrm{AP}$ in a saturated environment are considered, in view of varying data rates and packet sizes in an error free environment. Simulation results are presented and analyzed in the end.
\end{abstract}

\section{INTRODUCTION:}

There have been many developments in the field of networking in past decade but the most important one was the development of wireless networks that provides ease of feasibility to roam around. Wireless networks have been given a lot of attention because of its flexible nature in the data communication systems. It has been implemented as an extension or as an alternative of wired networks, which can be deployed virtually anywhere; from offices to university campus or providing hotspots at the airports, cafes, etc.

Besides having many advantages, wireless networks have some limitations as well which are generally due to the underlying medium being used. These limitations involve mobility, fewer battery life, frequent collisions and limited bandwidth. Such factors degrade the overall network performance [1].Many researchers have tried to increase the performance. To achieve this, many models were proposed to improve the performance by increasing the throughput and decreasing the delay, especially in a dense environment. Few of them achieved the desirable performance metrics with low delay and high throughput but most of them lack these desirable performance metrics.

The analysis of 802.11 CSMA/CA was first conducted by [2] to calculate the throughput in a saturated environment. Thereafter, many enhancements have been proposed in this area like Markov Chain model to incorporate retransmission without using the backoff process [3], to minimize the complexity and computation time the Markov chain model is reduced from two dimensions to one [4], etc. Another variation has been the calculation of packet drops probability, delay, etc. to analyze CSMA/CA based wireless network's

Comsats Institute of Information Technology, Islamabad Rifah International University, Islamabad. performance. But generally, the non-saturated cases are considered to emphasize on one parameter at a time.

In this study, authors have investigated the performance of a wireless network in terms of different data rates and achieved throughput, to extract the most suitable parameters for an infrastructure based wireless network in a saturated environment. The rest of the paper is organized as follows. Section II provides preliminaries and overview of some of the related concepts. Implementation details are discussed in section III. Simulation results and analysis are presented in section IV. Lastly, section V concludes the paper with the derived conclusion of this study and stating the future research directions.

\section{PRELIMINARIES}

\section{A. IEEE 802.11}

The main scope of this standard was to develop a set of specifications for connecting fixed, portables and mobile stations wirelessly within an area. It defined the Physical (PHY) and Media Access Control (MAC) layers for a wireless LAN. This standard was first proposed in 1997 by IEEE as IEEE Std. 802.11-1997. Currently the 802.11 family includes six over-the-air modulation techniques, all involving the same protocol. The most popular techniques of them are defined by $\mathrm{a}, \mathrm{b}$ and $\mathrm{g}$ amendments of the original standard, which are also known as 802.11 legacies. Security was included later in the $802.11 \mathrm{i}$ amendment. Recent amendment $802.11 \mathrm{n}$ is another modulation technique incorporating MIMO, but is still under development.

\begin{tabular}{|l|c|c|c|c|}
\hline Protocol & $\begin{array}{c}\text { Release } \\
\text { Date }\end{array}$ & $\begin{array}{c}\text { Op. Frequency } \\
\text { (GHz.) }\end{array}$ & $\begin{array}{c}\text { Data Rate } \\
\text { (Typical) }\end{array}$ & $\begin{array}{c}\text { Data Rate } \\
\text { (Max) }\end{array}$ \\
\hline Legacy & 1997 & $2.4-2.5$ & $1 \mathrm{Mbit} / \mathrm{s}$ & $2 \mathrm{Mbit} / \mathrm{s}$ \\
\hline $802.11 \mathrm{a}$ & 1999 & $\begin{array}{c}5.15-5.35 / 5.47-5.7 \\
25 / 5.725-5.875\end{array}$ & $25 \mathrm{Mbit} / \mathrm{s}$ & $54 \mathrm{Mbit} / \mathrm{s}$ \\
\hline $802.11 \mathrm{~b}$ & 1999 & $2.4-2.5$ & $6.5 \mathrm{Mbit} / \mathrm{s}$ & $11 \mathrm{Mbit} / \mathrm{s}$ \\
\hline $802.11 \mathrm{~g}$ & 2003 & $2.4-2.5$ & $25 \mathrm{Mbit} / \mathrm{s}$ & $54 \mathrm{Mbit} / \mathrm{s}$ \\
\hline $802.11 \mathrm{n}$ & 2006 & $2.4 \mathrm{GHz}$ or 5 & $200 \mathrm{Mbit} / \mathrm{s}$ & $540 \mathrm{Mbit} / \mathrm{s}$ \\
& $(\mathrm{draft})$ & & & \\
\hline
\end{tabular}

TABLE 1: SUMMARY OF STANDARDS IN 802.11 FAMILIES [13]

This family also includes other standards i.e. c, d, e, f, h and $\mathrm{j}$ which are mostly service enhancements and 
extensions or rather corrections of previously defined standards' specifications. First most adaptive and accepted standard was $802.11 \mathrm{~b}$ which was later followed by $802.11 \mathrm{a}$ and 802.11g [13]. A summary of the above discussed amendments is shown in table-1.

\section{B. Independent \& Infrastructure Basic Service Set}

In Independent BSS, or in simple terms ad-hoc network, STAs (stations) create direct communication with each other with out involving traffic channeling through a central controlling authority like access point (AP), but STAs must be in range of each other to communicate. It can accommodate small number of STAs for some specific purpose and it is formed for short period of time. The most common example of this type of network is a meeting or a conference, in which data is shared with in the conference room till the end of meeting or conference.

In contrast to IBSS / Ad-Hoc network, an Infrastructure Network (formally known as Infrastructure BSS) is established via APs. In this type all communication, including communication between STAs in same BSS, is done through APs. In infrastructure network STAs do not communicate directly they first have to send data to AP and then data is send from AP to destination STA. Figure-1(a), is an example of Independent BSS without any centralized controlling entity whereas Figure-1(b) represents an Infrastructure BSS.

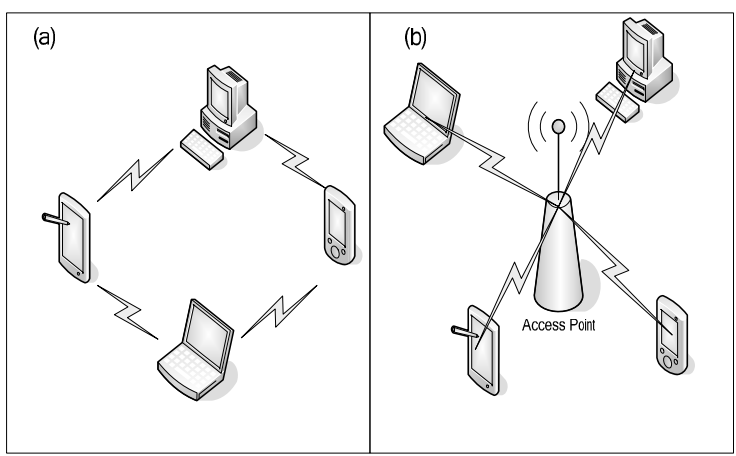

Figure 1: (a) Independent and (b) Infrastructure BSS architecture

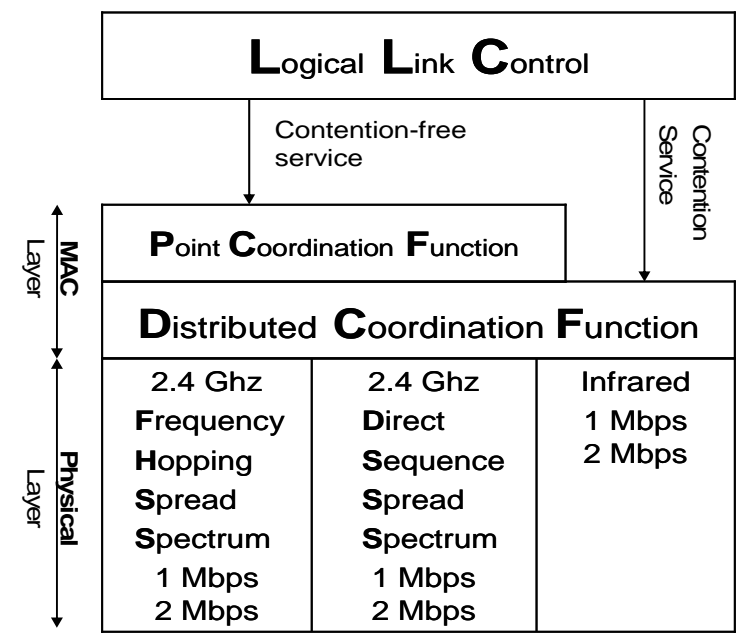

Figure-2: Protocol Layers' Architecture of Simple IEEE 802.11

\section{Distributed Coordination Function (DCF)}

IEEE 802.11 MAC provides distributed access along with optional centralized access to the medium, as shown in Figure-2. The distributed access control mechanism is called distributed coordination function (DCF) which is a random access scheme and depends upon the Carrier Sense Multiple Access with Collision Avoidance (CSMA/CA) [2]. As described in [5], in DCF before attempting to transmit data on the medium the station initially ensures that the medium is idle. A random back-off interval is selected by DCF, which is less than or equal to the current contention window $(\mathrm{CW})$ size based on the uniform distribution. When the medium is idle this back-off timer is decreased by one at every time slot, and it might have to wait for DIFS (DCF Inter-Frame space) following a successful transmission or EIFS in case of collision. If the medium is busy then the station will have to suspend its back-off timer until the transmission ends and the medium becomes idle. The station will transmit only when its back-off timer reaches zero. If there is a problem with the transmission, like it fails to reach or there is a collision, the station will invoke back-off procedure. To begin the back-off procedure first the size of contention window $(\mathrm{CW})$ is initialized with the initial value of CWmin, then this value is doubled till it reaches the maximum upper limit of $\mathrm{CW}$, i.e. CWmax. This value of CWmax will be kept until it is reset.

After this the station will set its back-off timer to a uniformly distributed random number in between interval of $[0, \mathrm{CW}]$ and then the station will retransmit when back-off timer reaches to zero. This procedure for retransmission will occur every time until the transmission is successful or transmission failure limit reaches its maximum value resulting in discarding of the packet, the value of $\mathrm{CW}$ will be reset to $\mathrm{CWmin}[6,7]$.

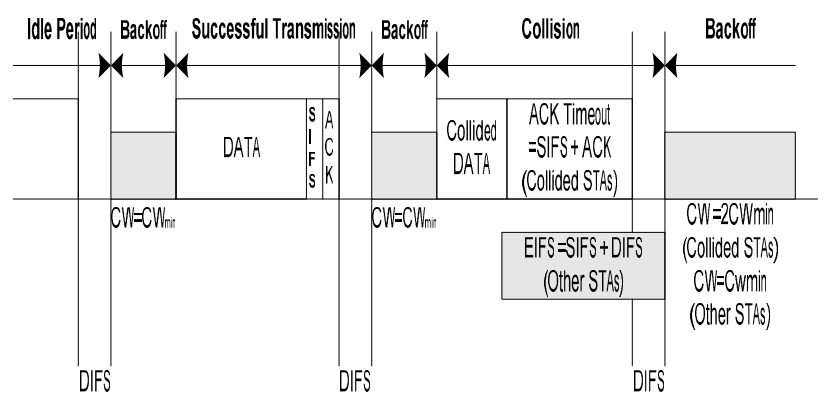

Figure-3: Basic Access Method

\section{Exponential Back-off Algorithm}

A packet transmitted first time has its $\mathrm{CW}$ set to $\mathrm{CWmin}$. After every collision the value of $\mathrm{CW}$ is doubled till it reaches CWmax. This process is called exponential back-off. The value of $\mathrm{CW}$ is not of fixed size because when station experiences a collision it has no idea that how many stations are involved collision. CW will be small if few numbers of packets collide then it will appropriate to choose random back-off time from a small set of small values. But CW will 
be long if large numbers of packets collide then there will a large set of values to choose a value for back-off timer $[5,8]$. See equation (1) below:

\section{Back - off Timer $=$ INT $(C W *$ Random ()$) *$ SlotTime}

(1)

where;

$\mathrm{CW}$, is an integer between CWmin and CWmax

Random ( ), is a random number generator

INT, is an integer function

If the set of values is small then choice to choose value for back-off timer will be limited. And if several STAs choose value from that set for back-off time then the probability of choosing same value will be high causing more collisions. To reduce the collision probability, time slots are defined in a way that STAs can determine that if another STA has started to transmit at the beginning of the previous time slot, then the risk time will be equal to time taken for packet transmission $[9,10]$. But if STAs are not capable to sense this, then the risk time will be double of packet transmission time. If STA has a packet to transmit and the medium remains idle longer than DIFS then this back-off algorithm is not used.

\section{E. DCF Throughput Analysis:}

The system throughput $\mathrm{S}$ can be defined as the fraction of time the channel is used to successfully transmit payload bits. If $\alpha$ is the back-off slot time per data frame transmission period, the time slot size will be equal to $\alpha$. Then the frame transmission period will be considered as the unit time and all other time intervals are normalized to this time unit. In order to explain this for finite number of stations, we assume that the system state alternates between two periods: Idle periods I, when stations have no frame to transmit and second is busy periods $B$ i.e. when at least one station transmits frame. Let $U$ be the time for useful transmission. If $E[X]$ denotes the expectation of a random variable $\mathrm{X}$, then the system throughput $\mathrm{S}$, as shown in equation (2) can be stated as:

$$
S=\frac{E(U)}{E(B)+E(I)}
$$

Equivalently, expresses the throughput as

$$
S=\frac{E[\text { Payload transmitted in a slot }]}{E[\text { Length of a slot time }]}
$$

When calculating the throughput, essential parameters are the durations when the medium becomes busy, because of successful transmission and unsuccessful transmission. As shown in Figure-4, durations of successful Tsucc and unsuccessful Tcol transmissions for Basic access methods is:
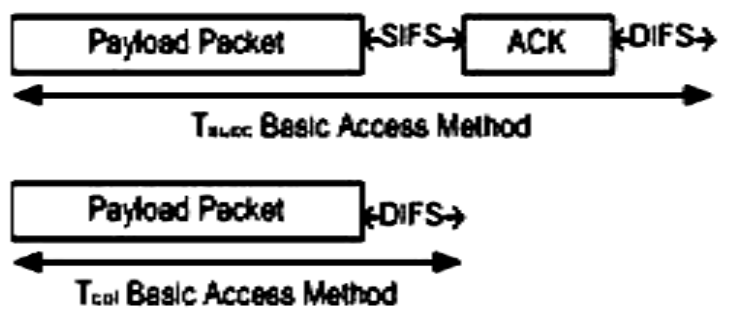

Figure-4: Tsucc \& Tcol for Basic

\section{IMPLEMENTATION DETAILS}

The purpose of this simulation is to analyze the performance of an infrastructure based wireless LAN. For simplicity it is considered that there is only one Basic Service Set (BSS) comprising of an Access Point (AP), which acts as a base station, and comprising of multiple number of nodes ranging in-between 1 to 40 .

It is also assumed that the nodes are in saturated mode i.e. every node has data to transmit all the time. This simulation will be utilizing Destination Sequenced Distance Vector (DSDV) as underlying network routing protocol and traffic will be generated with Constant Bit Rate (CBR) traffic source with data rate of 5.5 Mbps and $11 \mathrm{Mbps}$ (depending on the scenarios explained in next section). As there are two access methods of DCF to access the medium; Basic and RTS/CTS Access Method. This simulation will only be implementing Basic Access Method because both the RTS/CTS and Basic access method give the same results in case of a single BSS because there will be no hidden nodes.

\section{A. Factors Effecting Simulation}

There can me many factors which can effect the results of the simulation. These factors can be Simulation Runtime, Packet Size, Data Rate, and Radio Propagation Model. Each of the factors which according effect the simulation and how they are handled in simulation made in this paper.

Simulation Runtime: This factor does not affect a lot to the simulation. As it is perceived by running the simulation that the difference between long runtime and less runtime of the simulation is nearly negligible. By having long runtime it only takes long time to run the simulation and accumulate resources of the machine on which it is executed. Where as very less time can make the results unreliable because the starting time of the simulation (approx 2-5 sec) is warm-up time as in that time it initializes the connection and then after initializing the connections the simulation enters the steady state in which the results taken are more reliable.

Packet Size: According to protocols of link layer and transport layer rely on the checksum to detect the error in the packets. If erroneous bits are found in packets those packets are discarded. Keeping this in mind, in simulation it was observed that by having long packets the packet dropping rate was high which might be due to bit errors. And also due to long packet size the packet will be divided in more fragments which will cause more time for transmission which might leads to delay. On the other hand by having small packet size leads to higher proportional protocol header overhead. 
To cope with this factor effecting the simulation we have used two different sizes of packet size i.e. $512 \mathrm{~Kb}$ and $1024 \mathrm{~Kb}$. These packets according to understanding will provide reliable results as by using long or small packet sizes were leading to unreliable results in the simulation.

Data Rate: in IEEE 802.11b DCF, each competing node is given approximately equal opportunity for transmitting packets irrespective of time required to transmit a packet. So in this case if in a wireless network data rate and channel conditions are similar for each node and nodes have different packet sizes then the throughput and delay will be nearly same. For example if one node is transmitting at $1 \mathrm{Mbps}$ and other node is transmitting at $11 \mathrm{Mbps}$. As in the case of node transmitting at $1 \mathrm{Mbps}$ the time taken to transmit a frame will be longer as compared to the node transmitting at $11 \mathrm{Mbps}$, but the channel mostly will be used by the slower node. Hence the total throughput of both the nodes will be nearly same. In case of this project, IEEE $802.11 \mathrm{~b}$ support two different data rates i.e. 5.5 Mbps and $11 \mathrm{Mbps}$.

Simulation will be implementing both data rates to analyze both throughput and delay.

Radio Propagation Model: There are different radio propagation models, which can be implemented in NS-2 to simulate IEEE $802.11 \mathrm{~b}$ channel. In the simulation implemented, it is considered to have an open environment and range of nodes up to 100 meters, for implementing this Two Ray Ground model is used to implement in NS-2.

\section{B. Analyzing Traffic}

In this simulation, NS-2 generates new trace file format which is further utilized by the AWK script for calculating the Average Throughput and Delay for the current simulation scenario. As implemented in simulation AWK script calculates average throughput as follow.

$$
\text { Avg. Throughput }[\mathrm{Mbps}]=\frac{\text { recvdSize }}{(\text { stopTime }- \text { startTime })} \times \frac{8}{1000000}
$$

Here recvdSize is the total received packets' size, stopTime is the time when simulation ends and startTime is the starting time of the simulation. 8/1000000 is used to convert the throughput from bytes/second to Mbps. To calculate average delay, the script computes the time difference between the receive time and the send time for each packet and then divide the delay with the number of packets received to get average delay, as follow:

$$
\begin{aligned}
& \text { Delay }=\text { Delay }+(\text { recvTime }[\mathrm{i}]-\text { sendTime }[\mathrm{i}]) \\
& \text { Avg. Delay }[\mathrm{s}]=\text { Delay } / \text { recvdNum }
\end{aligned}
$$

Here ' $i$ ' represent the current packet. recTime and sendTime denotes receiving and sending time of the current' ' $\mathrm{i}$ ' packet. recvdNum is the total number of received. The delay computed is in seconds which is then converted to milliseconds.

\section{Simulation Scenarios}

This simulation is conducted in NS-2 network simulator $[11,12]$ and will comprise of two scenarios to understand the effect of packet size and data rate on performance of a simple wireless network. In simulation we will be having one BSS, error free channel and static configuration of nodes, so that all nodes are connected to base station. In BSS number of nodes will vary from 10 to 30 .

The simulation will run for 15 seconds and all nodes are in saturated state i.e. they have packets to transmit all the time during the simulation running time. To makes nodes saturated we have used CBR traffic generator which implements Poisson Arrival process of frames for transmission. And the nodes are modified to transmit data at a radius of $100 \mathrm{~m}$, to make this possible the transmission power of nodes is set to $281.8 \mathrm{~mW}$. The details provided above will be common for both scenarios discussed as follows:

Scenario 1: In this scenario the data rate will be kept constant, $11 \mathrm{Mbps}$, but packet size will vary i.e. $512 \mathrm{~Kb}$ or $1024 \mathrm{~Kb}$. Throughput and Delay will be recorded for both packet sizes, against the increasing number of nodes.

Scenario 2: This scenario will be involving fixed packet size of $512 \mathrm{~Kb}$. But in this scenario two different data rates will be utilized i.e. 5.5 Mbps or $11 \mathrm{Mbps}$. Number of nodes will be increasing same as that in Scenario 1, and throughput and delay will be recorded for both data rates.

\section{RESUlTS \& DISCUSSION}

The simulation was executed for all scenarios, and the results were calculated and recorded. The results are shown below for both throughput and delay calculated by implementing scenarios in the simulation.

\section{A. Throughput:}

The overhead of throughput in the DCF mode with basic access method is due to losses caused by the collisions and contention when the number of nodes in the wireless network increases, Figure-5 shows throughput when the data rate is kept same and the packet size is changed.

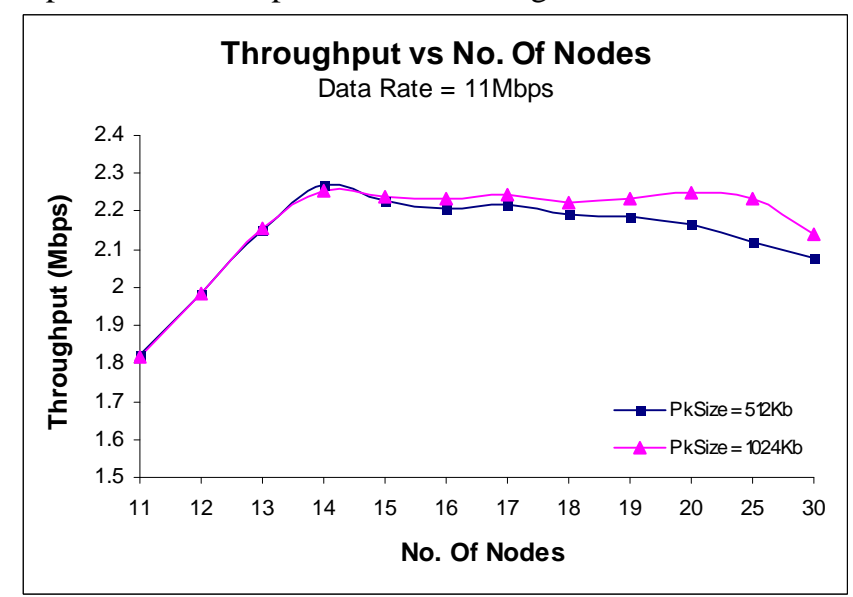

Figure-5: Throughput: Different Packet Sizes

Throughput stays nearly same to some number of nodes for both packet sizes. But when the number of nodes increases 
throughput gets slightly better for longer packet size. This is because the simulation is implemented by using an error free channel, thus longer sized packets makes the system more efficient. But this is not practically right, because in reality the channel is not error free and may experience fading of the channel due to obstacles, at that time longer packet sizes decease throughput.

Throughput is low for small packet size. This is because when packet size is small, transmissions will complete fast causing channel to be available very abruptly. So the nodes will try to acquire the channels causing high contention, thus the throughput will decrease.

However, if the packet size is kept same but the data rate is changed. From Figure- 6 it is evident that the throughput is high when data rate is high. This is because when data rate is high and number of nodes increases, the channel gets more utilized; thus causing throughput to increase until it becomes steady. Whereas, in the case of low data rate, the throughput is comparatively lower than the high data rate when large number of nodes is considered. This is because when the data rate is low and number of nodes is high more packets will be dropped. This drop in throughput is caused because nodes will have to wait longer, then they have to wait in high data rata, to access the channel which will lead to dropping of packets and collision; as basic access method is taken into consideration.

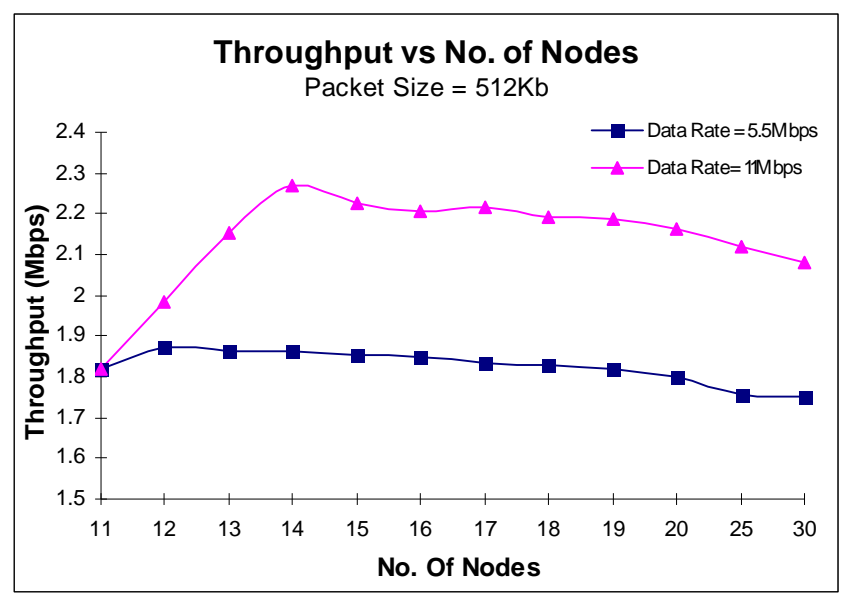

Figure-6: Throughput: Different Data Rates

B. Delay:

As discussed above, the effect of packet size and data rate on the throughput of the wireless network simulated. There is another factor which is critical while analyzing the performance of a network, known as delay. The figure below represents the effect on delay when we have fixed data rate but variable packet size.

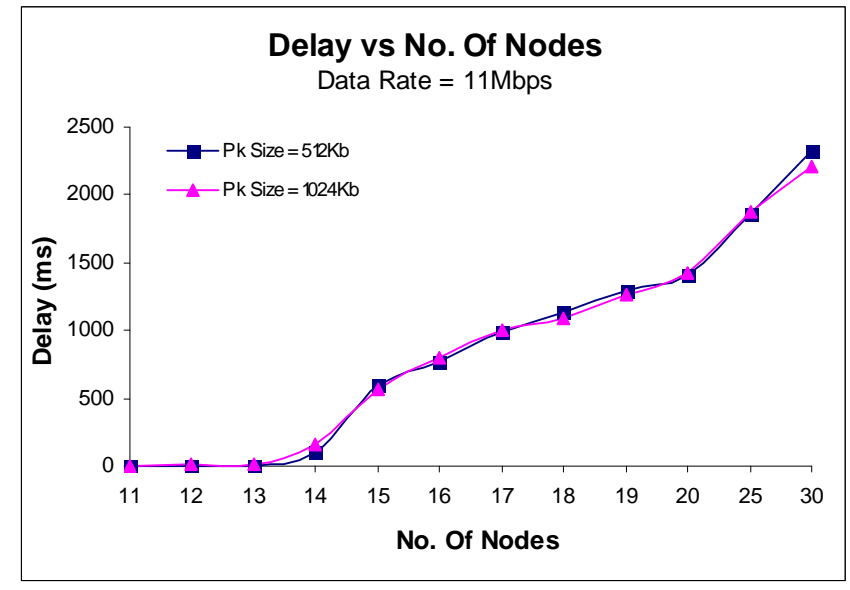

Figure-7 Delay: Different Packet Size

In Figure-7 it is observed that delay for small packet and long packet size is nearly same. The reason behind is that when we have small packet size the transmission of packets will be fast but the contention for the channel will be high which will cause more delay. Whereas in large packets the transmission time for the packets will be high causing more waiting by the contending nodes to access the channel and might also cause more packet dropping.

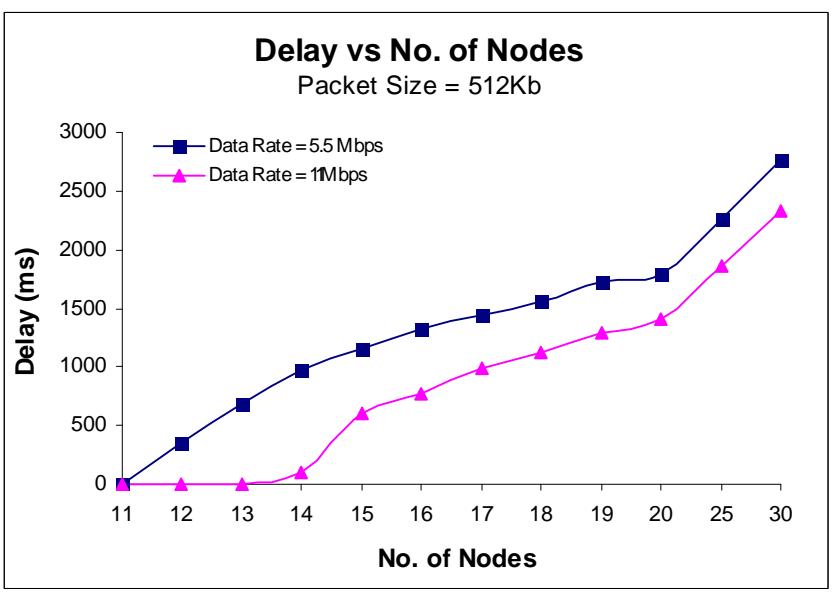

Figure-8 Delay: Different Data Rates

Figure- 8 shows the graph for delay plotted against the number of nodes, with same packet size but different data rates. It is observed in the graph above that the delay increases very rapidly when data rate is low and the number of nodes keep on increasing. This is because when data rate is low, time to transmit packets will be high causing channel to stay busy and make other nodes to wait till the transmission is complete. Whereas if data rate is high, then transmission of packets will be fast which will make channel available, thus the delay will be low. But there will be time when number of nodes transmitting packets will be high and it will become impossible for channel to accommodate packets transmitted from those nodes. This will make waiting time by the contending nodes high and collision, resulting in increased delay but still comparatively lower than low data rate.

So this can assume from the results that to have less delay 
in the network we should use high data rate regardless of packet size.

\section{CONCLUSION}

In this paper a simulation model to compute the throughout and delay performance of IEEE $802.11 \mathrm{~b}$ Distributed Coordinated Function (DCF) is presented. This simulation model assumes to have a finite number of saturated stations (nodes) transmitting packets on an ideal channel and uses Basic Access Method for acquiring channel for transmission in a single BSS. This model is validated by comparing its results with other analytical and simulated models.

The evaluated results show that the performance of basic access method used for IEEE 802.11 mainly depends upon the number of stations in the wireless network. It is also evaluated that by using small packet size the contention for channel between the stations becomes higher causing high delay and if small packets are transmitted upon low data rate the bandwidth overhead results in reduction in the throughput However, on the other hand by having longer or small packet size and high data rate with large number of nodes, results in more channel utilization that causes high throughput and low delay on the overall performance of the network improve. In future, authors intend to increase the number of APs so that RTS/CTS mechanism can also be incorporated which does not differ with basic access method in case of a single AP; as considered in this study.

\section{REFERENCE:}

[1] Gast, Mathew S 2005, "802.11 Wireless Networks: The Definitive Guide", O’ Reilly, Sebastopol, California, USA

[2] Bianchi, G 2000, "Performance analysis of the IEEE 802.11 distributed coordination function", IEEE Journal on Selected Areas in Communications.

[3] Ziouva, E \& Antonakopoulos, T 2002, "The effect of finite population on IEEE 802.11 wireless LANs throughput/delay performance", in The 2002 Mediterranean Electro- technical Conference, Melecon 2002, Egypt.

[4] Vukovic, I., Smavatkul, N. 2004, "Delay Analysis of Different Backoff Algorithms in IEEE 802.11",in Proceeding of IEEE Vehicular Technology Conference

[5] IEEE 1999, "Standard for Wireless LAN Medium Access Control (MAC) and Physical Layer (PHY) specifications", ISO/IEC 8802-11: 1999(E).

[6] Zhai, Y K \& Fang Y 2004, "Performance of Wireless LANs Based on IEEE 802.11 MAC Protocols", Wiley Inter-Science.

[7] Bianchi, G, Fratta, Luigi \& Oliveri, Matteo 1996, "Performance evaluation and enhancements of the CSMA/CA MAC protocol for 802.11 wireless LANs", Proc. PIMRC, Tapei, Taiwan.

[8] Godfrey Tan, John Guttag, 2004 "Time-based Fairness Improves Performance in Multi-rate WLANs", MIT Computer Science and Artificial Intelligence Laboratory.

[9] Jari Korhonen, Ye Wang, 2005, "Effect of Packet Size on Loss Rate and Delay in Wireless Links", School of Computing, National University of Singapore, Singapore.

[10] Kim ,J. H. and Lee, J. K., 1999 "Capture effects of wireless CSMA/CA protocols in rayleigh and shadow fading channels," IEEE Trans. Veh. Tech., vol. 48, no. 3, pp. 1277--1286, Mar 1999.

[11] Fall, K., Varadhan, K., 2006 "The ns Manual, (formerly ns Notes and Documentation)", The VINT Project: a collaboration between researchers at UC Berkeley, LBL, USC/ISI and Xerox PARC

[12] Eitan Altman, Tania Jiménez, 2003 "NS Simulator for beginners", Univ. de Los Andes, Merida, Venezuela

[13] IEEE 802.11 (March 25, 2009). Available at Wikipedia: http://en.wikipedia.org/wiki/IEEE 802.11 